\title{
Evaluación de la avifauna de la ciudad de La Paz, Bolivia
}

\author{
Avifauna evaluation of the La Paz City, Bolivia
}

\author{
Omar Martínez, Manuel Olivera, Carmen Quiroga e Isabel Gómez
}

Museo Nacional de Historia Natural, Universidad Mayor de San Andrés, Casilla 8706, La PazAolivia. E-mail Omar Martínez: o martinez25@hotmail.com
Presentado: $\quad 08 / 01 / 2010$ 23/07/2010 Publicado online: $14 / 12 / 2010$

\section{Resumen}

Desde el año 1996 al 2003, mediante métodos de observación directa, caminatas y capturas con redes niebla, realizamos un inventario y obtuvimos datos sobre la historia natural, gremios tróficos y patrones de migración de la avifauna de la ciudad de La Paz (Bolivia). Registramos 136 especies de aves en 30 localidades de estudio. Las familias más representativas fueron Tyrannidae, Emberizidae y Furnariidae con 19, 16 y 15 especies, respectivamente. Registramos 18 especies de aves acuáticas y dos especies (Tachuris rubrigastra y Phleocryptes melanops) de passeriformes especialistas de totorales. Dos especies de furnáridos (Cranioleuca henricae y Upucerthia harterti) fueron endémicas bolivianas. Detectamos 57 especies en alguna categoría de migración. Movimientos altitudinales inusuales fueron observados para cinco especies (e.g. Pitangus sulphuratus, Pyrocephalus rubinus). Diecinueve especies fueron consideradas raras (e.g. Lesbia nuna, Poospiza boliviana). Los insectívoros y frugi-granívoros son los gremios tróficos mas representativos entre comunidades. El valle de la ciudad de La Paz es una ruta obligatoria para muchas aves migrantes latitudinales (boreales y australes) y altitudinales, que provienen del Altiplano por el oeste y las especies de Yungas por el este.

Palabras clave: Aves, biodiversidad, aves migrantes, Andes, Bolivia.

\section{Abstracts}

Since 1996 to 2003, we realized an inventory and obtained data on the natural history, trophic guilds and migration patterns of the avifauna of La Paz (Bolivia), based on direct observation, random walks and captures with mist nets. A total of 136 species of birds in 30 localities of study were registered. Tyrannids, Emberizids and Furnariids were the families most representatives with 19, 16 and 15 species, respectively. Eighteen species of waterbirds and two species of passerine rush (called totorales) specialists as Tachuris rubigastra and Phleocryptes melanops, were registered. Two furnariids species (Cranioleuca henricae and Upucerthia harterti) were Bolivian endemics. We detected 57 species in some category of migrants. Unusual elevational movements were observed for five species (e.g. Pitangus sulphuratus, Pyrocephalus rubinus). Nineteen species were considered rare (e.g. Lesbia nuna, Poospiza boliviana). Insectivores and frugi-granivores were the trophic guilds most representatives between communities. The La Paz City Valley is one obligatory route for many latitudinal (neartic and austral) and altitudinal migrants birds, which arise from highland by the western and species of mountain forest (called Yungas) by the eastern.

Keywords: Birds, biodiversity, migrants birds, Andes, Bolivia.

\section{Introducción}

En el Neotrópico, los bosques secos se distribuyen de forma disyunta, en el oeste de América Central (desde México hasta Costa Rica), en el norte de Venezuela y Colombia, noreste de Brasil, sudoeste de Ecuador y Perú, el Gran Chaco y regiones adyacentes de Sudamérica Central (Argentina, Bolivia, Brasil, Paraguay) y en los valles desde Venezuela hasta el norte de la Argentina (Herzog \& Kessler 2002). En Bolivia, los bosques secos ubicados entre 1500-3200 m de altitud (López 2003), son distribuidos extensivamente en las tierras bajas del sur del país, extendiéndose dentro de los Andes en los valles semihúmedos (Ribera et al. 1996, Herzog \& Kessler 2002). Los valles secos del norte de Bolivia en el cual se incluyen el valle de la ciudad de La Paz, son conocidos como islas de hábitat dentro de una matriz generalmente de puna y altiplano.

La hoya de la ciudad de La Paz y los valles adyacentes comprenden un complejo mosaico de ambientes antropogenizados y naturales, donde procesos de colonización de las plantas en los espacios libres de asfalto entre calles, edificaciones y campos abiertos, permiten el establecimiento de comunidades vegetales denominadas ruderales (García 1991, 1997), las cuales albergan comunidades de aves tolerantes a la presencia humana; mientras que en los alrededores y zonas alejadas del centro existen ambientes menos intervenidos con mayor diversidad de plantas nativas. En el valle de la ciudad de La Paz, existe un marcado gradiente altitudinal entre la región norte (La Cumbre) con
4600 m, Centro de La Paz (3600 m) y la zona Sud (Tahuapalca) con $2600 \mathrm{~m}$, reflejando algunos patrones de recambio en las comunidades aviares.

El presente trabajo actualiza el conocimiento sobre la composición específica de la avifauna, realiza un análisis de los gremios tróficos y los patrones de migración de las comunidades de aves terrestres y acuáticas del valle de la ciudad de La Paz y zonas circundantes.

\section{Materiales y métodos}

\section{Área de estudio}

El estudio se realizó en 30 localidades de la ciudad de La Paz, Provincia Murillo, Departamento de La Paz, Bolivia (Fig. 1, Tabla 1). El clima se caracteriza por tener una estación árida prolongada (7-8 meses) y una estación húmeda corta (4-5 meses), las temperaturas más altas se registran en la zona sur $\left(2{ }^{\circ} \mathrm{C}\right.$ más que el centro de la ciudad) y las más bajas hacia el norte (La Cumbre), también la precipitación y la humedad disminuyen en sentido norte-sur, hasta llegar a la zona de los valles secos (Mecapaca, El Palomar) al sur de área de estudio. El promedio total anual de temperaturas máximas en 50 años fue de $17,2{ }^{\circ} \mathrm{C}$ y el total anual de lluvias en 80 años fue de 579,1 mm (Segaline Nieto \& Cabré 1988).

La vegetación de la hoya de La Paz, esta caracterizada por formaciones arbustivas como Dunalia brachyacantha (Solanac.), Malva parviflora (Malvac.), Sisymbrium irio (Crucif.), Mutisia 


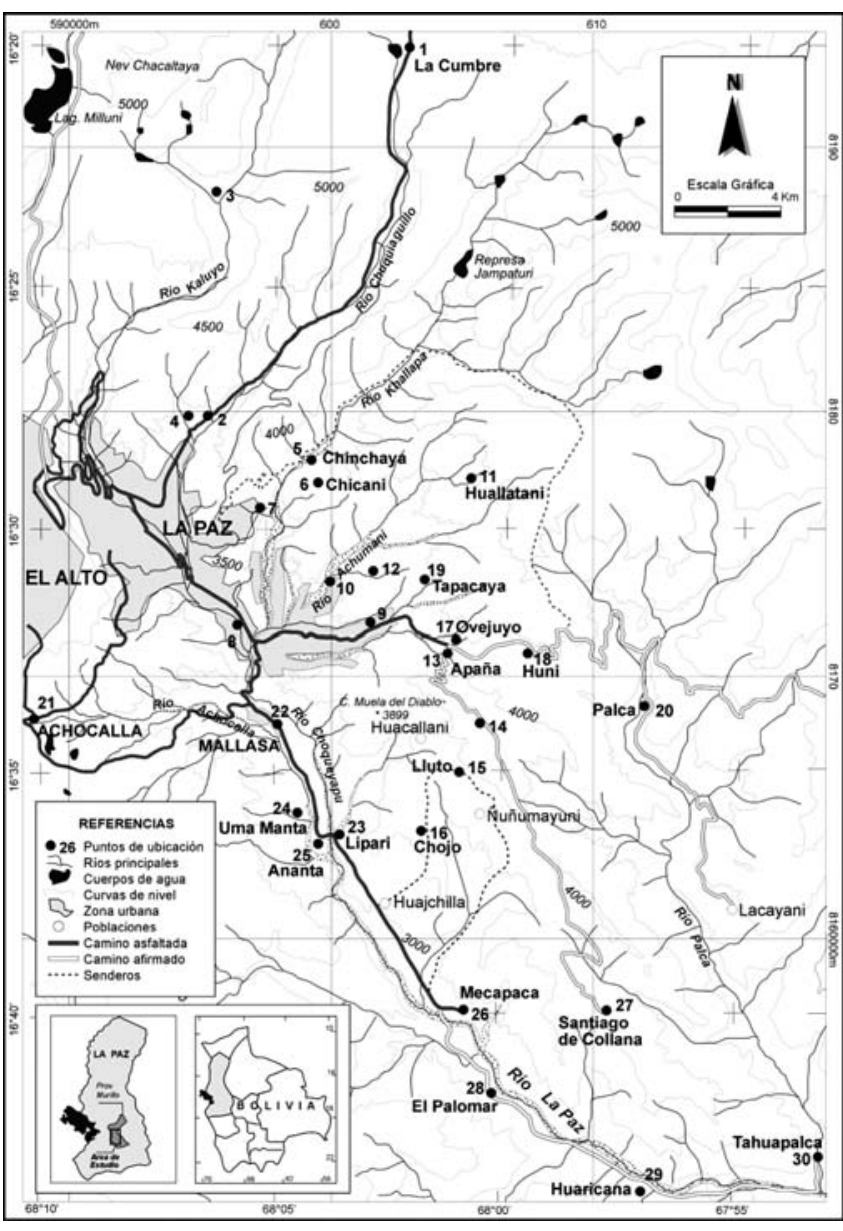

Figura 1. Mapa de ubicación de las localidades de estudio en el valle de la ciudad de La Paz, Bolivia.

acuminata (Composit.) (García 1997). En los lechos de río es común Cortaderia sp. (Gram.). En los valles secos se encuentran arbustos como Adesmia miraflorensis, Atriplex semibaccata (Chenopod.), semiarbóreos como Nicotiana glauca (Solanac.), Tecoma arequipensis (Bignoniac.) y árboles nativos como Prosopis laevigata (Mimosac.) e introducidos como Eucalyptus globulus (Myrtac.) y Pinus radiata (Pinac.) (García 1991).

El crecimiento del área urbana de la ciudad de La Paz, se concentra en la zona norte-sur, con proyecciones y tendencias hacia los valles secos del sur de la hoya.

\section{Muestreo de la avifauna}

Durante ocho años (1996-2003), realizamos 76 salidas (seis de ellas de cuatro días de trabajo de campo) a diferentes localidades de la ciudad de La Paz. La composición de la avifauna fue determinada a partir de observaciones directas. Realizamos también el registro de llamadas y cantos mediante la técnica de play back. Capturamos aves en tres localidades (Ananta, Mecapaca y Collana) con redes niebla y anillamos las aves con anillos de metal.

La descripción de la dieta se basó en observaciones sobre el comportamiento de forrajeo y los hábitos alimenticios de las aves y la literatura (Fjeldså \& Krabbe 1990, Del Hoyo et al. 1992, 1994, 1996, 1997, 1999, 2001, 2002, 2003, 2004, 2005, Isler \& Isler 1987). Las categorías de los grupos tróficos fueron: 1) insectívoros (especies que primariamente se alimentan de insectos y otros artrópodos); 2) frugi-granívoros (especies cuyo ítem alimenticio principal son frutos, granos, semillas y otros materiales vegetales); 3) nectarívoros (especies que se alimentan de néctar); 4) carnívoros (especies que se alimentan de presas vertebradas vivas) y 5) especies con otras dietas (omnívoros, carroñeros e ictiófagos) (Herzog \& Kessler 2002).

Las selecciones del hábitat se basaron en los siguientes criterios: 1) especies típicas de bosques secos (Herzog y Kessler, 2002), 2) especies de otras formaciones no boscosas, hábitats de puna y matorrales altoandinos y 3) especies de formaciones artificiales de bosques de eucaliptos, áreas urbanas y periurbanas. Realizamos caminatas, cubriendo todos los lugares posibles para monitorear las aves, complementar inventarios y recabar datos sobre los grupos tróficos de las comunidades de aves.

Finalmente, realizamos un análisis sobre patrones de migración de las aves, considerando: 1) migrantes parciales, aquellas especies descritas para el continente según mapas de distribución (Rappole et al. 1983, Stotz et al. 1996) y 2) migrantes estrictos según reportes locales (Jahn et al. 2001, Hennessey et al. 2003).

\section{Resultados y discusión}

\section{Composición taxonómica de las comunidades de aves}

Un total de 136 especies de aves terrestres y acuáticas fueron registradas en todos los sitios combinados (Apéndice 1), distribuidas en 37 familias (16 paserinos y 21 no paserinos), de las cuales Tyrannidae (22 especies; 16\%), Emberizidae (19; 15\%) y Furnariidae $(17 ; 12,5 \%)$, fueron las de mayor número de especies, las mismas contribuyen altamente al número típico de especies de valles secos encontrados en otros estudios (Fjeldså y Mayer 1996, Martínez 2000, Herzog \& Kessler 2002). Veintisiete especies de aves (e. g. Upucerthia harterti, Gnorimopsar chopi) fueron nuevos registros para el valle de La Paz con respecto a Ribera (1991). Así mismo, tres especies son nuevos registros (Anas puna, Asthenes sclateri, Diglossa sittoides) según Remsen y Traylor (1989) y dos nuevas para La Paz (Hirundo rustica, Gnorimopsar chopi) con relación a Hennessey et al. (2003).

De las 14 especies endémicas y amenazadas de Bolivia (Sagot 1998, Hennessey et al. 2003), dos (Upucerthia harterti, Cranioleuca henricae) fueron registradas como raras en nuestros sitios. Upucerthia harterti es considerada casi amenazada y asociada a los valles interandinos entre 1450-2960 m (Sttatersfield et al. 1998); fue observada sólo en dos oportunidades (mayo y octubre de 1997) en Mecapaca. En ésta localidad, fue registrada también C. henricae por Hennessey (1997), por MO en marzo del 2000, y en febrero de 2009 por OM, se trata de un furnárido endémico valluno (Maijer \& Fjeldså 1997), está asociada al matorral alto xerofítico espinoso dominado por Prosopis laevigata, tunales (Opuntia ficus-indica) y molles (Schinus mollis), actualmente $C$. henricae es considerada como en peligro de extinción en Bolivia (Rocha \& Balderrama 2009). Poospiza boliviana, casi endémica de valles secos (Sagot 1998; Stattersfield et al. 1998), fue considerada rara, con pocos registros en quebradas y asociado a cardonales (Trichocereus sp.) con parches de Prosopis laevigata y matorrales xerofíticos espinosos de Adesmia miraflorensis y Opuntia ficus indica. En cuanto a los endemismos zoogeográficos (Stotz et al. 1996), registramos 25 especies (e.g. Asthenes humilis, Idiopsar brachyurus, ver Apéndice 1) de los Andes Centrales denotados con CAN por Hennessey et al. (2003).

Durante seis campañas (entre 1998 y 1999) en tres localidades (Ananta, Collana y Mecapaca) capturamos 153 aves terrestres, de las cuales 130 fueron anilladas. Phytotoma rutila, fue la especie 
Tabla 1. Localidades de estudio en el valle de la ciudad de La Paz (Bolivia).

\begin{tabular}{|c|c|c|c|c|}
\hline$\#$ & Localidad & Coordenadas & Altitud (m) & Piso ecológico \\
\hline 1 & Cumbre & $16^{\circ} 20^{\prime} \mathrm{S}-68^{\circ} 02^{\prime} \mathrm{W}$ & 4600 & Altoandino superior \\
\hline 2 & Chuquiaguillo & $16^{\circ} 27^{\prime} \mathrm{S}-68^{\circ} 06^{\prime} \mathrm{W}$ & 3800 & Altoandino superior e inferior \\
\hline 3 & Kaluyo & $16^{\circ} 23^{\prime} \mathrm{S}-68^{\circ} 06^{\prime} \mathrm{W}$ & 4100 & Altoandino inferior y superior \\
\hline 4 & Pura Pura & $16^{\circ} 27^{\prime} \mathrm{S}-68^{\circ} 06^{\prime} \mathrm{W}$ & 3800 & Altoandino inferior \\
\hline 5 & Chinchaya & $16^{\circ} 09^{\prime} \mathrm{S}-68^{\circ} 04^{\prime} \mathrm{W}$ & 3400 & Puna \\
\hline 6 & Chicani & $16^{\circ} 29^{\prime} \mathrm{S}-68^{\circ} 04^{\prime} \mathrm{W}$ & 3500 & Puna \\
\hline 7 & Khallapa & $16^{\circ} 29^{\prime} \mathrm{S}-68^{\circ} 05^{\prime} \mathrm{W}$ & 3600 & Puna \\
\hline 8 & Seguencoma & $16^{\circ} 31^{\prime} \mathrm{S}-68^{\circ} 05^{\prime} \mathrm{W}$ & 3100 & Puna \\
\hline 9 & Cota Cota & $16^{\circ} 32^{\prime} \mathrm{S}-68^{\circ} 03^{\prime} \mathrm{W}$ & 3400 & Puna - Valle seco \\
\hline 10 & Achumani & $16^{\circ} 31^{\prime} \mathrm{S}-68^{\circ} 04^{\prime} \mathrm{W}$ & 3300 & Puna - Valle seco \\
\hline 11 & Huallatani & $16^{\circ} 29^{\prime} \mathrm{S}-68^{\circ} 01^{\prime} \mathrm{W}$ & 3700 & Puna \\
\hline 12 & Lomas del Sur & $16^{\circ} 31^{\prime} \mathrm{S}-68^{\circ} 03^{\prime} \mathrm{W}$ & 3600 & Puna \\
\hline 13 & Villa Apaña & $16^{\circ} 32^{\prime} \mathrm{S}-68^{\circ} 01^{\prime} \mathrm{W}$ & 3850 & Puna \\
\hline 14 & Cerro Cuñamani & $16^{\circ} 34^{\prime} \mathrm{S}-68^{\circ} 00^{\prime} \mathrm{W}$ & 4367 & Altoandino inferior \\
\hline 15 & Lluto & $16^{\circ} 35^{\prime} \mathrm{S}-68^{\circ} 01^{\prime} \mathrm{W}$ & 3850 & Puna \\
\hline 16 & Chojo & $16^{\circ} 36^{\prime} \mathrm{S}-68^{\circ} 02^{\prime} \mathrm{W}$ & 3850 & Puna \\
\hline 17 & Ovejuyo & $16^{\circ} 31^{\prime} \mathrm{S}-68^{\circ} 01^{\prime} \mathrm{W}$ & 4500 & Altoandino inferior y puna \\
\hline 18 & Huni & $16^{\circ} 32^{\prime} \mathrm{S}-67^{\circ} 57^{\prime} \mathrm{W}$ & 3800 & Altoandino inferior y puna \\
\hline 19 & Tapacaya & $16^{\circ} 31^{\prime} \mathrm{S}-68^{\circ} 01^{\prime} \mathrm{W}$ & 4000 & Altoandino inferior y puna \\
\hline 20 & Palca & $16^{\circ} 34^{`} \mathrm{~S}-67^{\circ} 57^{\prime} \mathrm{W}$ & 4500 & Altoandino inferior y puna \\
\hline 21 & Achocalla & $16^{\circ} 34^{\prime} \mathrm{S}-68^{\circ} 10^{\prime} \mathrm{W}$ & 3700 & Puna \\
\hline 22 & Mallasa & $16^{\circ} 34^{\prime} \mathrm{S}-68^{\circ} 10^{\prime} \mathrm{W}$ & 2800 & Valle seco interandino \\
\hline 23 & Lipari & $16^{\circ} 37^{\prime} \mathrm{S}-68^{\circ} 04^{\prime} \mathrm{W}$ & 3400 & Valle seco interandino \\
\hline 24 & Uma Manta & $16^{\circ} 36^{`} \mathrm{~S}-68^{\circ} 4.5^{\prime} \mathrm{W}$ & 2750 & Valle seco interandino \\
\hline 25 & Ananta & $16^{\circ} 37^{\prime} \mathrm{S}-68^{\circ} 03^{\prime} \mathrm{W}$ & 2700 & Valle seco interandino \\
\hline 26 & Mecapaca & $16^{\circ} 40^{\prime} \mathrm{S}-68^{\circ} 01^{\prime} \mathrm{W}$ & 2840 & Valle seco interandino \\
\hline 27 & Santiago de Collana & $16^{\circ} 40^{\prime} \mathrm{S}-67^{\circ} 57^{\prime} \mathrm{W}$ & 3800 & Altoandino inferior y puna \\
\hline 28 & El Palomar & $16^{\circ} 41^{\prime} \mathrm{S}-68^{\circ} 00^{\prime} \mathrm{W}$ & 2800 & Valle seco interandino \\
\hline 29 & Huaricana & $16^{\circ} 29^{\prime} \mathrm{S}-67^{\circ} 05^{\prime} \mathrm{W}$ & 2650 & Valle seco interandino \\
\hline 30 & Tahuapalca & $16^{\circ} 45^{\prime} \mathrm{S}-67^{\circ} 57^{\prime} \mathrm{W}$ & 2600 & Valle seco interandino \\
\hline
\end{tabular}

más común en las capturas, representando el 26\% (particularmente en Ananta). Otras especies frecuentemente capturadas fueron, Catamenia analis (13\%), Columbina picui (13\%, ésta sólo en Mecapaca) y Psilopsiagon aymara con el 7\% (Tabla 2). Un total de 31 especies (12 familias) fueron representadas en las capturas. En Collana, capturamos en una sola oportunidad al tiránido (Muscisaxicola maculirostris) y los furnáridos (Phacellodomus striaticeps y Asthenes sclateri [punensis]). En general, las recapturas fueron pocas (7), pero todas en los mismos sitios donde fueron capturados inicialmente, lo cual parece indicar cierto grado de fidelidad por ciertos territorios.

Tabla 2. Número de capturas de las diez especies más comúnmente capturadas en tres localidades (Ananta, Mecapaca y Collana) del valle de La Paz, Bolivia.

\begin{tabular}{lcc}
\hline Especie & $\begin{array}{c}\text { Individuos } \\
\text { capturados }\end{array}$ & \% de la muestra \\
\hline Phytotoma rutila & 39 & 25,49 \\
Columbina picui & 19 & 12,5 \\
Catamenia analis & 19 & 12,5 \\
Psilopsiagon aymara & 11 & 7,24 \\
Asthenes dorbignyi & 10 & 6,58 \\
Sicalis olivascens & 9 & 5,88 \\
Upucerthia andaecola & 5 & 3,27 \\
Sappho sparganura & 4 & 2,61 \\
Thraupis bonariensis & 3 & 1,97 \\
Carduelis xanthogastra & 3 & 1,97 \\
\hline
\end{tabular}

Ribera (1991) registró 13 especies de aves acuáticas para el valle de La Paz, con sólo tres anátidos (Chloephaga melanoptera, Anas flavirostris y A. georgica) presentes. Nosotros registramos 18 especies, con siete especies de anátidos (e.g. Anas cyanoptera, Oxyura jamaicensis), cinco de las cuales se encuentran en las lagunas Charan y Jaccha Kkota de Achocalla (ver Apéndice 1). Estas lagunas se caracterizan por la presencia de cinturones de totora (Schoenoplectus californicus tatora), donde 12 especies residentes de aves acuáticas (e.g. Fulica ardesiaca, Pardirallus sanguinolentus) y cinco visitantes (e. g. Phalacrocorax brasilianus, Egretta thula) fueron registradas. Algunas especies de amplia distribución en tierras bajas tienen poblaciones moderadamente bien definidas en la zona de la puna: A. cyanoptera, P. sanguinolentus, Gallinula chloropus, Phleocryptes melanops, Tachuris rubrigastra, y Chrysosomus thilius (Fjeldså 1985). Todas ellas presentes en el valle de La Paz con poblaciones moderadas, excepto $C$. thilius, no registrado en la hoya.

Algunas especies de aves, se encuentran en los límites del área del valle de La Paz y son localmente poco comunes hasta raras, entre ellas destacamos a Idiopsar brachyurus y Poospiza torquata. I. brachyurus, conocido sólo en pocas localidades en la puna alta del este de los Andes desde Perú al norte de la Argentina, fue registrado en los límites del valle de La Paz: Río Choquekkota a $9 \mathrm{~km}$ del desvío del camino Calacoto-Palca, camino a la Mina San Francisco, 4150 m (Remsen et al. 1982). En una oportunidad, capturamos una pareja de I. brachyurus (31 de mayo de 1998) en un bosque de Polylepis pepei y Gynoxis sp. 
Tabla 3. Número de especies y porcentaje total de la muestra en los grupos tróficos de aves del valle de la ciudad de La Paz.

\begin{tabular}{lcc}
\hline Grupos tróficos* & $\begin{array}{c}\text { Número de } \\
\text { especies }\end{array}$ & Porcentaje del total \\
\hline Insectívoros & 55 & 40,4 \\
Frugi-granívoros & 42 & 30,9 \\
Carnívoros & 10 & 7,3 \\
Nectarívoros & 8 & 5,9 \\
Otras dietas: & 12 & \\
Omnívoros & 6 & 8,8 \\
Ictiófagos & 3 & 4,4 \\
Carroñeros & 136 & 2,2 \\
\hline Total & & 100 \\
\hline
\end{tabular}

en Pongo, Provincia Murillo, La Paz (4200 m). OM registró, $I$. brachyurus en las cotas altas (3900-4100 m) de Palca en abril de 2002. El único registro de $P$. torquata fue en mayo de 1999 en Tahuapalca, en el límite sur del valle de La Paz. No se conoce mucho sobre el estado actual de Cinclus leucocephalus, que al parecer se encuentran mejor conservadas en el nordeste de la cuenca, hacia el Río Palca, incluyendo las lagunas de Huallatani, Chojña Khota y los ríos Tabaco y Huancapampa (localidad de Tapacaya en este estudio).

\section{Gremios tróficos}

Un aspecto ecológico que marca la heterogeneidad de las comunidades de aves y sus hábitats está relacionada con factores ambientales, principalmente la variación altitudinal (2700-4100 $\mathrm{m})$ que parece incidir notoriamente en la composición de los gremios tróficos del Valle de La Paz. De todas las especies de aves, $55(40,4 \%)$ fueron insectívoras, 42 (30,9\%) frugi-granívoras, 10 $(7,3 \%)$ carnívoras, $8(5,9 \%)$ nectarívoras y 21 (15,4\%) tienen otras dietas (e.g. omnívoros, carroñeros, ictiófagos) (Tabla 3).

Realizamos un análisis comparativo de los gremios tróficos entre los sitios estudiados. El resultado refleja que los insectívoros y frugi-granívoros tienen las más altas proporciones entre comunidades. Insectívoros variaron desde $7 \%$ en Palca a $65 \%$ en Chojo y frugi-granívoros desde 16\% en Achocalla hasta 59\% en Huaricana (Tabla 4). La proporción de carnívoros varío desde 4\% (Chuquiaguillo) hasta un 17\% (Huni), nectarívoros desde 5\% (Callapa, Cuñamani y Achocalla) a 20\% (Chuquiaguillo) y aquellas especies con otras dietas desde 3\% (Chinchaya, Achumani, Apańa y Ovejuyo) a 45\% (Achocalla) (Tabla 4). Herzog y Kessler (2002) determinaron que un 39\% de la avifauna de Mecapaca fue insectívora y un 35\% fueron frugi-granívoros. En nuestro estudio, los insectívoros y frugi-granívoros de Mecapaca representaron el 44 y 37\%, respectivamente. De las especies típicas de bosques secos, 28 (41\%) son insectívoras, 26 (38\%)

Tabla 4. Número de especies $(\mathrm{N})$ por categoría trófica y porcentaje (entre paréntesis) de representatividad en la muestra en 30 localidades del Valle de La Paz, Bolivia.

\begin{tabular}{|c|c|c|c|c|c|c|c|c|}
\hline \multirow{2}{*}{ Localidades } & \multirow{2}{*}{ Insec } & \multirow{2}{*}{ F-gra } & \multirow{2}{*}{ Carni } & \multirow{2}{*}{ Necta } & \multicolumn{3}{|c|}{ Otras dietas } & \multirow{2}{*}{$\mathrm{N}$} \\
\hline & & & & & Omni & Ictio & Carro & \\
\hline 1. Cumbre & $1(10)$ & $4(40)$ & $2(20)$ & $1(10)$ & $1(10)$ & $0(0)$ & $1(10)$ & 10 \\
\hline 2. Chuquiaguillo & $6(24)$ & $12(48)$ & $1(4)$ & $5(20)$ & $0(0)$ & $0(0)$ & $1(4)$ & 25 \\
\hline 3. Kaluyo & $4(40)$ & $4(40)$ & $0(0)$ & $1(10)$ & $1(10)$ & $0(0)$ & $0(0)$ & 10 \\
\hline 4. Pura Pura & $3(21)$ & $8(58)$ & $1(7)$ & $2(14)$ & $0(0)$ & $0(0)$ & $0(0)$ & 14 \\
\hline 5. Chinchaya & $15(38)$ & $16(41)$ & $2(5)$ & $5(13)$ & $0(0)$ & $0(0)$ & $1(3)$ & 39 \\
\hline 6. Chicani & $7(47)$ & $3(20)$ & $2(13)$ & $2(13)$ & $0(0)$ & $0(0)$ & $1(7)$ & 15 \\
\hline 7. Callapa & $5(27)$ & $11(58)$ & $1(5)$ & $1(5)$ & $1(5)$ & $0(0)$ & $0(0)$ & 19 \\
\hline 8. Següencoma & $5(42)$ & $5(42)$ & $0(0)$ & $1(8)$ & $1(8)$ & $0(0)$ & $0(0)$ & 12 \\
\hline 9. Collana & $14(34)$ & $17(41)$ & $3(7)$ & $5(12)$ & $0(0)$ & $0(0)$ & $2(5)$ & 41 \\
\hline 10. Achumani & $12(41)$ & $10(35)$ & $2(7)$ & $4(14)$ & $0(0)$ & $0(0)$ & $1(3)$ & 29 \\
\hline 11. Huallatani & $6(46)$ & $4(31)$ & $2(15)$ & $1(8)$ & $0(0)$ & $0(0)$ & $0(0)$ & 13 \\
\hline 12. Lomas del Sur & $8(36)$ & $9(42)$ & $1(4)$ & $3(14)$ & $0(0)$ & $0(0)$ & $1(6)$ & 22 \\
\hline 13. Apaña & $14(47)$ & $13(43)$ & $0(0)$ & $2(7)$ & $0(0)$ & $0(0)$ & $1(3)$ & 30 \\
\hline 14. Cuñamani & $11(55)$ & $7(35)$ & $0(0)$ & $1(5)$ & $0(0)$ & $0(0)$ & $1(8)$ & 20 \\
\hline 15. Lluto & $3(38)$ & $4(50)$ & $0(0)$ & $1(12)$ & $0(0)$ & $0(0)$ & $0(0)$ & 8 \\
\hline 16. Chojo & $11(65)$ & $5(29)$ & $0(0)$ & $0(0)$ & $0(0)$ & $0(0)$ & $1(6)$ & 17 \\
\hline 17. Ovejuyo & $10(36)$ & $10(36)$ & $3(11)$ & $4(14)$ & $0(0)$ & $0(0)$ & $1(3)$ & 27 \\
\hline 18. Huni & $4(33)$ & $5(42)$ & $2(17)$ & $0(0)$ & $0(0)$ & $0(0)$ & $1(8)$ & 12 \\
\hline 19. Tapacaya & $5(42)$ & $4(34)$ & $1(8)$ & $1(8)$ & $0(0)$ & $0(0)$ & $1(8)$ & 12 \\
\hline 20. Palca & $1(7)$ & $9(60)$ & $3(20)$ & $2(13)$ & $0(0)$ & $0(0)$ & $0(0)$ & 15 \\
\hline 21. Achocalla & $11(29)$ & $6(16)$ & $2(5)$ & $2(5)$ & $11(29)$ & $5(13)$ & $1(3)$ & 39 \\
\hline 22. Mallasa & $13(33)$ & $13(33)$ & $3(7)$ & $7(17)$ & $3(7)$ & $1(3)$ & $0(0)$ & 40 \\
\hline 23. Lipari & $11(38)$ & $15(52)$ & $0(0)$ & $3(10)$ & $0(0)$ & $0(0)$ & $0(0)$ & 29 \\
\hline 24. Uma Manta & $3(22)$ & $5(39)$ & $0(0)$ & $5(39)$ & $0(0)$ & $0(0)$ & $0(0)$ & 13 \\
\hline 25. Ananta & $15(37)$ & $16(40)$ & $2(5)$ & $3(7.5)$ & $3(7.5)$ & $0(0)$ & $1(3)$ & 40 \\
\hline 26. Mecapaca & $28(44)$ & $24(37)$ & $3(5)$ & $5(8)$ & $0(0)$ & $2(3)$ & $2(3)$ & 62 \\
\hline 27. Cota Cota & $21(41)$ & $18(35)$ & $3(6)$ & $5(10)$ & $3(6)$ & $0(0)$ & $1(2)$ & 50 \\
\hline 28. El Palomar & $10(48)$ & $8(38)$ & $1(4)$ & $2(10)$ & $0(0)$ & $0(0)$ & $0(0)$ & 22 \\
\hline 29. Huaricana & $5(29)$ & $10(59)$ & $1(6)$ & $0(0)$ & $0(0)$ & $0(0)$ & $1(6)$ & 16 \\
\hline 30. Tahuapalca & $5(23)$ & $12(54)$ & $1(5)$ & $4(18)$ & $0(0)$ & $0(0)$ & $0(0)$ & 22 \\
\hline
\end{tabular}

Insec = Insectívoros, F-gra = Frugi-granívoros, Carni = Carnívoros, Necta = Nectarívoros, Omni = Omnívoros, Ictio = Ictiófagos, Carro = Carroñeros y N = Número de especies. 
frugi-granívoras, siete (10\%) nectarívoros, seis (9\%) carnívoros y un carroñero (Phalcoboenus megalopterus). De las especies típicas de la puna y cabecera de valle, $32(50 \%)$ son frugi-granívoras, $20(31 \%)$ insectívoras, siete $(11 \%)$ carnívoras, tres $(5 \%)$ nectarívoras y dos tienen otras dietas (Tabla 4).

Los insectívoros y frugi-granívoros fueron más comunes en bajas elevaciones; los nectarívoros parecen tener la misma pauta, aunque ciertas especies frecuentan tierras altas (e.g. Oreotrochilus estella, Diglossa carbonaria), pero en general son más comunes en pequeñas islas de hábitat (e.g. parches de Nicotiana, Tecoma, Salvia). Ciertas aves suelen frecuentar plantaciones de eucalipto (Eucalyptus sp.) para alimentarse de diferentes estructuras de estos árboles (botones florales, flores, frutos verdes). En el bosque de eucaliptos de Pura Pura (50 Has), encontramos 14 especies de aves en los diferentes estratos; nectarívoros como O. estella y $D$. carbonaria, que también forrajean en flores de eucalipto durante la época seca. En estos bosques son frecuentes algunas aves (e.g. T. sayaca, T. bonariensis) exclusivistas del dosel. En el interior de estos bosques y asociados a otros matorrales encontramos otras aves (e.g. Troglodytes aedon, Catamenia analis). Algunos carnívoros (e.g. Falco femoralis, F. sparverius) utilizan como perchas las ramas altas de estos árboles.

Los carnívoros fueron más comunes en ciertos ambientes antrópicos como el basurero de Mallasa. Un patrón muy común en varios sitios andinos es la ausencia de carnívoros en bosques secos (Herzog \& Kessler 2002). Nuestras observaciones parecen seguir esta pauta. Algunos carnívoros de amplia distribución y de hábitats generalistas (e.g. Falco sparverius) y especies de hábitats no boscosos (e.g. Buteo polyosoma) se extienden a lo largo del gradiente en el valle de La Paz, así como Geranoaetus melanoleucus, observado con poca frecuencia alrededor de los boques de eucalipto de Mallasa. En la categoría de otras dietas (omnívoros, carroñeros, ictíofagos), sobresalen los omnívoros que variaron desde 3\% en Mecapaca hasta un 29\% en Achocalla. Entre los escasos registros de carroñeros, destacamos Vultur gryphus, en la ribera del Río La Paz (Mecapaca) y otro reciente en noviembre de 2009 en la cima $(3650 \mathrm{~m})$ de Llacasa (parte alta de Huajchilla-Taipichullo), se trata de una especie vulnerable (Vul) en Bolivia (Balderrama et al. 2009).

Los ictíofagos tienen una proporción relativamente baja (13\%) en Achocalla (Tabla 4). Visitantes estacionales de las lagunas de Achocalla como las garzas (cuatro especies) y el cormorán (Phalacrocorax brasilianus) son exclusivamente de hábitos ictiófagos. El zambullidor (Rollandia rolland) considerado migrante austral, aunque con una población local residente fue observado en varias oportunidades atrapando peces (Orestias sp.) en las lagunas de Achocalla.

Entre los nectarívoros, sobresalen los colibríes (5 especies), aunque otras aves, como Diglossa carbonaria y D. sittoides, suelen perforar flores tubulares (e.g. la "kantuta", Cantua buxifolia y Tecoma arequipensis). D. carbonaria, aparentemente se extiende hacia zonas mas altas (Ovejuyo, Apaña, Cerro Cuñamani, Chuquiaguillo), aunque también suele visitar áreas periurbanas y jardines como en Cota Cota y plazas centrales de la ciudad de La Paz. D. sittoides está restringido a hábitats poco intervenidos de valles secos como en Mecapaca. Algunos nectarívoros parecen tener preferencia por flores específicas en parches de hábitats. Colibri coruscans tiene marcada preferencia por Mutisia acuminata (Composit.) en Tamboraque $(3010 \mathrm{~m})$, a $99 \mathrm{~km}$ al este de Lima, Perú (Hainsworth 1977). En nuestro estudio, $M$. acuminata fue frecuentado por C. coruscans y Patagona gigas; aunque $C$. coruscans forrajea también en Dunalia brachyacantha (Solanac.). Murcia (1987), encontró flores de Fuchsia sp. (Onagrac.), visitadas la mayor parte de día por Diglossa albilatera en la Finca Merenberg (2300 m), Huila, Colombia. En áreas urbanas de La Paz, es común observar Diglossa carbonaria en flores de Fuchsia sp. y kantuta (Cantua buxifolia), en estas mismas plantas forrajea también C. coruscans y Sappho sparganura.

\section{Patrones de migración y especies visitantes}

Un total de 57 especies de aves fueron detectadas en alguna categoría de migración, de las cuales 26 fueron migrantes (22 australes y 4 boreales) estrictos. De las 55 especies migrantes boreales registradas en Bolivia (Gómez \& Aguilar 1998), sólo detectamos seis, todas con poblaciones que se reproducen en el Neotrópico. Además, dos especies (e.g. Bubulcus ibis y Cathartes aura) fueron migrantes boreales parciales en el valle de La Paz (Anexo 1). Coccyzus americanus fue registrado en Cota Cota (9 de diciembre de 2002), ésta puede migrar hasta Bolivia durante ciertas estaciones del año (Rappole et al. 1983). Falco peregrinus (migrante boreal y austral) fue citado para bajas y altas altitudes de Santa Cruz, Cochabamba y Oruro (Remsen \& Ridgely 1980). Dott (1985) registró $F$. peregrinus en dos oportunidades en la Laguna Alalay (Cochabamba a $2450 \mathrm{~m}$ de altitud), en diciembre de 1969 y abril de 1975. En nuestro estudio, F. peregrinus fue observado solo en cuatro oportunidades (junio de 1996 en Achocalla, mayo de 1998 en Santiago de Collana, junio de 1998 en Mallasa y otro individuo fue observado cruzando el Río Achocalla en mayo de 1999). Dott (1985) reportó la presencia de la golondrina migrante boreal Hirundo rustica en Bolivia para la Laguna Alalay (Cochabamba) en varias oportunidades entre 1974 y 1975. En nuestro estudio, un grupo de 10 individuos de esta golondrina fue observado (21 de octubre de 2002) en la laguna Charan Khota de Achocalla.

Por otra parte, 22 especies fueron migrantes australes estrictos (e.g. Tyrannus savana, Turdus amaurochalinus) y 29 parciales (e.g. Phalacrocorax brasilianus, Caprimulgus longirostris) (Apéndice 1). T. savana fue registrado en varias oportunidades entre noviembre y diciembre del 2002 en Cota Cota frecuentando las plantaciones de eucalipto (Eucalyptus globulus) y percheando en Salix babylonica. Ribera (1991) menciona también un avistamiento único en Cota Cota (1986) pero no indica la época para hacer comparaciones con nuestros datos. P. brasilianus y Nycticorax nycticorax se congregan y reproducen en hábitats de tierras altas en la zona de la puna y no tiene diferenciación morfológica en sus poblaciones andinas (Fjeldså 1985). P. brasilianus fue observado en dos ocasiones en la laguna Jaccha Kkota (febrero 2001 y septiembre 2003) y una vez en la laguna Charan Khota (febrero de 2002) y $N$. nycticorax es visitante estacional en las lagunas de Achocalla y Cota Cota. C. longirostris fue observado al atardecer del 29 de marzo de 1998, en una quebrada hacia el río Achocalla (en la desembocadura del Río La Paz) cerca Umamanta (Jupapina) en hábitats de tierras malas (badlands).

Mediante capturas detectamos 18 especies (e.g. Falco sparverius, Anairetes parulus) migrantes, entre ellas a F. sparverius (31 de mayo de 1998) en Ananta al filo del bosque de Prosopis y una terraza (badlands), en la red se encontró también su presa una lagartija (Liolaemus sp.). Por otra parte, 20 especies migrantes altitudinales fueron registradas en el valle de La Paz, 
tres de ellas también consideradas migrantes por Hennessey et al. (2003), como Ardea alba, registrada (18 de febrero de 2003 y 10 de julio de 2005) en la laguna Charan Kkota (Achocalla). El picaflor (Lesbia nuna) fue considerado raro debido a un solo registro en Umamanta en un fragmento de Nicotiana glauca, aparentemente su distribución sería más sureńa, puesto que registramos la especie en Caracato, al Sur del Valle del río La Paz. Recientemente, OM detecto un territorio de L. nuna a $3650 \mathrm{~m}$ en Llacasa en la parte alta de Ananta.

En el Chaco boliviano, 44 especies de migrantes australes fueron registradas por capturas (Jahn et al. 2002). Comparado con nuestro estudio, siete especies (Columbina picui, Camptostoma obsoletum, Elaenia albiceps, Pitangus sulphuratus, Turdus amaurochalinus, Zonotrichia capensis y Thraupis sayaca) fueron compartidas en ambos sitios. Un total de 51 especies propias del Cono Sur, están presentes en alguna estación anual en el valle de La Paz. Muchas de estas especies tienen poblaciones residentes en La Paz (e.g. Turdus amaurochalinus, Saltator aurantiirostris). Los tiranos, C. obsoletum, E. albiceps, Pyrocephalus rubinus y $P$. sulphuratus fueron considerados visitantes ocasionales en La Paz. Un macho solitario de P. rubinus, fue observado (24 de julio de 2003) percheando en eucalipto al borde de un campo de cultivo y la laguna de Achocalla. Fjeldså y Krabbe (1990) mencionan que $P$. rubinus asciende hasta $3050 \mathrm{~m}$ en el Perú y $2500 \mathrm{~m}$ al sur del mismo país. En Bolivia, P. sulphuratus se distribuye hasta los $3400 \mathrm{~m}$ (Hennessey et al. 2003). Una pareja de P. sulphuratus fue observada (febrero de 1971) en el nido, construido en un árbol de eucalipto cerca de la ciudad de Cochabamba a $2550 \mathrm{~m}$ (Dott 1984). En La Paz, P. sulphuratus fue registrado el 17 de julio y el 27 de septiembre de 2003 en la laguna de Achocalla $(3700 \mathrm{~m})$. P. sulphuratus; frecuentemente capturaba insectos acuáticos y pequeños peces (Orestias sp.), en pleno vuelo. Los hábitos ictiófagos de P. sulphuratus han sido descritos en ciertas lagunas de Mendoza, Argentina (Richard, 1986).

El rango de expansión de aves introducidas en Bolivia, ha sido poco descrito. El gorrión Passer domesticus fue introducido en la Argentina en 1872, Brasil en 1903 y Chile 1904 (Dott 1986). Esta especie ha sido registrada en el sur de Bolivia desde al menos 1936 (Bond \& Meyer de Schauensee 1942, Serrano $\&$ Cabot 1983). Dott ha descrito ampliamente la expansión de Passer domesticus en Bolivia, cuyos primeros registros fueron descritos para Villa Montes en 1930 (Eisentraut 1935; citado en Dott 1986). Desde 1969 a 1975, P. domesticus fue registrado en altitudes bajas, medias y pocos registros en áreas de la puna y altiplano, incluyendo la ciudad de La Paz y Oruro, aunque fueron considerados no comunes (Dott 1986). En nuestro estudio, detectamos $P$. domesticus en Mecapaca $(2700 \mathrm{~m})$ y en pequeños grupos (3-5 individuos) y grandes grupos 15-20 individuos.

\section{Conservación}

Muchas aves tropicales y/o de tierras mas bajas son comercializadas en la ciudad de La Paz y El Alto, en particular los loros (e.g. Amazona aestiva, Ara auricollis). No obstante, la ocurrencia de algunas especies tropicales en el valle de La Paz, sería producto de la huída de estos animales. Uno de estos casos sería el tordo (Gnorimopsar chopi), el cual fue registrado por vez primera en 1997, ésta ave se ha adaptado notablemente a los bosques de eucalipto de Cota Cota (Quiroga et al. 1998a, 1998b) y frecuenta los alrededores de Calacoto y otros barrios de la zona sur de La Paz como el zoológico de Mallasa.
Por otra parte, el relativo alto número de especies (57), registradas en alguna categoría de migración y aproximadamente 17 especies visitantes altitudinales en la hoya de La Paz, nos sugiere que el valle constituye una ruta obligatoria para muchas aves durante sus desplazamientos. Por esta razón, es necesario estudiar las comunidades de aves para poder interpretar el impacto en sus poblaciones por efecto del progresivo avance de las zonas urbanas fundamentalmente hacia los valles secos del Sud, agravado por las constantes pérdidas de bosquecillos naturales con vegetación nativa (e.g. Prosopis, Nicotiana), importantes recursos naturales para el forrajeo de las aves residentes y visitantes estacionales. Considerando también dos especies endémicas ( $U$. harterti y una de ellas en peligro: $C$. henricae), dos amenazadas ( $V$. gryphus, $C$. henricae), una propia del área endémica para aves (EBA 055) y cuatro especies para la EBA 056, sumado a la pérdida de ciertos tipos de hábitat como las bromelias y arbustos espinosos en los badlands (véase por ejemplo, Tarifa et al. 2004), que son arrasados para urbanizar terrenos. Por lo tanto, la conservación de la avifauna del valle de La Paz se basa en el entendimiento de su historia natural.

\section{Agradecimientos}

A Jon Fjeldså del Zoological Museum-University of Copenhagen (Denmark) por la consecución de fondos y apoyo al proyecto. A Susan E. Davis del Museo de Historia Natural Noel Kempff Mercado por proporcionarnos los anillos para el monitoreo. Al Museo Nacional de Historia Natural y la Colección Boliviana de Fauna (La Paz, Bolivia) por permitirnos la revisión de especimenes y la bibliografía. A los miembros del Club ProAves "Jamachis": Lawrence Rubey, Victor Bullen, Brian Woods, Ian Rossell, Mike Stuart, Bo Westman, Douglas Mason y Omar Rocha por su apoyo logístico en varias salidas. A Nick Acheson de Oxford University (England) por la revisión y aportes al manuscrito. A Enrique Richard de EcoDreams (Bolivia) por los datos de P. sulphuratus y sus aportes bibliográficos. Sebastian K. Herzog de Armonía-BirdLife International (Bolivia) revisó y contribuyó a mejorar el manuscrito. A todos los compañeros biólogos que de manera desinteresada nos acompańaron en las salidas de campo (Esther López, Gabriela Villegas, Hugo Araníbar, Andrea Montoya, Jan Ohlson, Cinthya Jurado, Andrea Dávila, Faviany Lino, María Luz Menacho, Eddy Pérez, Gabriela Flores, Boris Ríos, Andrea Morales, Heidy López, Erika de la Gálvez, Carlos Uzquiano, Yorema Gutiérrez, Álvaro Uzquiano, Isabel Moya y Luís Arteaga).

\section{Literatura citada}

Balderrama, J.A., C. Quiroga, O. Martínez \& M. Crespo. 2009. Vultur gryphus. In: L. F. Aguirre, R. Aguayo, J. Balderrama, C. Cortez y T. Tarifa, eds. Libro rojo de la fauna silvestre de vertebrados de Bolivia. Ministerio de Medio Ambiente y Agua. La Paz, Bolivia. pp. 363-364.

Bond, J. \& R. Meyer de Schauensee. 1942. The birds of Bolivia. Part I. Proc. Acad. Nat. Sci. Philad. 94: 307-391.

Del Hoyo J., A. Elliott \& J. Sargatal. 1992. Handbook of the birds of the world. Vol. 1: Ostrich to Ducks. Lynx Edicions, Barcelona.

Del Hoyo J., A. Elliott \& J. Sargatal. 1994. Handbook of the birds of the world. Vol. 2: New World Vultures to Guineafowl. Lynx Edicions, Barcelona.

Del Hoyo J., A. Elliott \& J. Sargatal. 1996. Handbook of the birds of the world. Vol. 3: Hoatzin to Auks. Lynx Edicions, Barcelona. 
Del Hoyo J., A. Elliott \& J. Sargatal. 1997. Handbook of the birds of the world. Vol. 4: Sandgrouse to Cuckoos. Lynx Edicions, Barcelona.

Del Hoyo J., A. Elliott \& J. Sargatal. 1999. Handbook of the birds of the world. Vol. 5: BarnOwls to Hummingbirds. Lynx Edicions, Barcelona.

Del Hoyo J., A. Elliott \& J. Sargatal. 2001. Handbook of the birds of the world. Vol. 6: Mousebirds to Hornbills. Lynx Edicions, Barcelona.

Del Hoyo J., A. Elliott \& J. Sargatal. 2002. Handbook of the birds of the world. Vol. 7: Jacamars to woodpeckers. Lynx Edicions, Barcelona.

Del Hoyo J., A. Elliott \& D. Christie. 2003. Handbook of the birds of the world. Vol. 8: Broadbills to Tapaculos. Lynx Edicions, Barcelona.

Del Hoyo J., A. Elliott \& D. Christie. 2004. Handbook of the birds of the world. Vol. 9: Cotingas to Pipits. Lynx Edicions, Barcelona.

Del Hoyo J., A. Elliott \& D. Christie. 2005. Handbook of the birds of the world. Vol. 10: Cuckoo-shrikes to Thrushes. Lynx Edicions, Barcelona.

Dott H. E. M. 1984. Range extensions, one new record, and notes on winter breeding of birds in Bolivia. Bulletin British Ornithological Club 104 (3): 104-109.

Dott H. E. M. 1985. North american migrants in Bolivia. Condor 87: 343-345.

Dott H. E. M. 1986. The spread of the House Sparrow Passer domesticus in Bolivia. Ibis 128: 132-137.

Fjeldså J. 1985. Origin, evolution, and status of the avifauna of the Andean wetlands. In: P.A. Buckley, M. S. Foster, E. S. Morton, R. S Ridgely \& F. G. Buckley, eds.. Neotropical Ornithology. Ornithological monographs 36. Pp. 85-112.

Fjeldså J. \& N. Krabbe. 1990. Birds of the high Andes. Zoological Museum. University of Copenhagen, Denmark. 876 p.

Fjeldså J. \& S. Mayer. 1996. Recent ornithological surveys in the Valleys region, southern Bolivia - and the possible role of Valleys for the evolution of the Andean avifauna. DIVA Technical Report 1. Ronde.

García E. 1991. Flora de la ciudad de La Paz. In: E. Forno \& M. Baudoin, eds. Historia Natural de un valle en los Andes: La Paz. Instituto de Ecología-Universidad Mayor de San Andrés. La Paz, Bolivia. Pp. 151-168.

García E. 1997. Composición florística y ecología de las comunidades ruderales de las calles de la ciudad de La Paz. Ecología en Bolivia 29: 1-18.

Gómez I. \& M. S. Aguilar. 1998. Aves migratorias neárticas en Bolivia. Aves y conservación en Bolivia 1: 92-97.

Hainsworth F. R. 1977. Foraging efficiency and parental care in Colibri coruscans. Condor 79: 69-75.

Hennessey A. B. 1997. Mecapaca. In: A. B. Hennessey, ed. Aves en Bolivia $\mathrm{N}^{\mathrm{o}} 1$. Nov. 97. Boletín semestral bilingüe de Armonía. Santa Cruz, Bolivia. Pp. 11

Hennessey A. B., S. K. Herzog \& F. Sagot. 2003. Lista anotada de las aves de Bolivia. Asociación Armonía/BirdLife International. Santa Cruz de la Sierra, Bolivia. 238 p.

Herzog S. K. \& M. Kessler. 2002. Biogeography and composition of dry forest bird communities in Bolivia. Journal für Onithologie 143: 171-204.

Isler M. L. \& P. R. Isler. 1987. The Tanagers. Natural History, Distribution and Identification. Smithsonian Institution Press, Washington, D.C.

Jahn A. E., S. E. Davis \& A. M. Saavedra Z. 2002. Patrones en la migración austral de aves entre temporadas y hábitats en el Chaco boliviano, con notas de observaciones y una lista de especies. Ecología en Bolivia 37 (2): 31-50.

López R. P. 2003. Diversidad florística y endemismo de los valles secos bolivianos. Ecología en Bolivia 38 (1): 27-60.
Maijer S. \& J. Fjeldså. 1997. Description of a new Cranioleuca spinetail from Bolivia and a "leap frog pattern" of geographic variation in the genus. Ibis 139: 606-616.

Martínez O. 2000. Avifauna de los bosques montanos de El Palmar (Provincia Zudañez, Departamento Chuquisaca). Documentos Ecología en Bolivia 6: 1-14.

Murcia C. 1987. Estructura y dinámica del gremio de colibríes (Aves: Trochilidae) en un bosque andino. Humboldtia 1: 29-64.

Quiroga C., M. Olivera \& O. Martínez. 1998a. Registros ornitológicos destacables de 1996-1997 en el Valle de La Paz, Prov. Murillo, Dpto. La Paz. Aves y Conservación en Bolivia 1: 112-117.

Quiroga C., M. Olivera, O. Martínez, H. Araníbar, I. Gómez \& A. Uzquiano. 1998b. Primer registro del pájaro negro Gnorimopsar chopi para el Valle de La Paz. Aves en Bolivia 2: 16-17.

Rappole J. H., E. S. Morton, T. E. Lovejoy, III \& J. L. Ruos. 1983. Neartic avian migrants in the Neotropics. U. S. Deparment of the Interior Fish and Wildlife Service. World Wildlife Fund - U. S. 664 p.

Remsen J. V. Jr. 1985. Community organization and ecology of birds of high elevational humid forest of the Bolivian Andes. In: P.A. Buckley, M.S. Foster, E.S. Morton, R. S. Ridgely \& F. G. Buckley, eds. Neotropical Ornithology. Ornithological Monographs 36. Pp. 733-756.

Remsen J. V., Jr \& R. S. Ridgely. 1980. Additions to the avifauna of Bolivia. Condor 82: 69-75.

Remsen J. V., Jr., T. A. Parker, III \& R. S. Ridgely. 1982. Natural history notes on some poorly known Bolivian birds. Le Gerfaut 72: 77-87.

Remsen J.V., Jr \& M.A. Traylor Jr. 1989. An annotated list of the birds of Bolivia. Buteo Books. Vermilion, South Dakota. $79 \mathrm{p}$.

Ribera M. O. 1991. Aves. In: E. Forno \& M. Baudoin, eds. Historia Natural de un Valle en Los Andes: La Paz. Instituto de Ecología-UMSA. La Paz, Bolivia. Pp. 345-420.

Ribera M. O., M. Liberman, S. Beck \& M. Moraes. 1996. Vegetación de Bolivia. In: K. Mihotek, ed. Comunidades, territorios indígenas y biodiversidad en Bolivia. Santa Cruz, Bolivia. Pp. 169-222.

Richard E. 1986. Predación del benteveo sobre peces y tortugas. Nuestras Aves 4 (11): 3-4.

Rocha, E. \& J. A. Balderrama. 2009. Aves. Pp. 305-417 In: L. F. Aguirre, R. Aguayo, J. Balderrama, C. Cortez y T. Tarifa, eds. Libro rojo de la fauna silvestre de vertebrados de Bolivia. Ministerio de Medio Ambiente y Agua. La Paz

Sagot F. 1998. Aves endémicas, amenazadas y mal protegidas de Bolivia. Aves y conservación en Bolivia 1: 64-67.

Segaline Nieto H. \& R. Cabré. 1988. El clima de La Paz. Datos del observatorio San Calixto. La Paz, Bolivia. 80 p.

Serrano P. P. \& N. J. Cabot. 1983. Passer domesticus, nueva especie para Bolivia. Acta Vertebrata Doñana 10: 212-213.

Stattersfield A. J., M. J. Crosby, A. J. Long. \& D. C. Wege. 1998. Endemic birds areas of the world. Priorities for biodiversity conservation. BirdLife International. Cambridge, UK.

Stotz D. F., J. W. Fitzpatrick, T. A. Parker III \& D. K. Moskovits. 1996. Neotropical birds. Ecology and conservation. The University of Chicago. Chicago.

Tarifa T., F. Fontúrbel, D. Achá, J. Rodriguez, C. Molina, M. C. López, M. R. Baudoin, C. Buitrón, A. Canseco, M. García, Y. Higueras, D. Kopp, J. Pacajes, P. Romecin \& V. Urrelo. 2004. Vizcachas (Lagidium viscacia, Chinchillidae) en hábitats fragmentados en la ciudad de La Paz y sus alrededores: bases para su conservación. Ecología en Bolivia 39(1): 53-74. 
Apéndice 1. Lista de las aves del la ciudad de La Paz y regiones aledañas. Localidades: 1. Cumbre, 2. Chuquiaguillo, 3. Kaluyo, 4. Pura Pura, 5. Chinchaya, 6. Chicani, 7. Callapa, 8. Seguencoma, 9. Collana, 10. Achumani, 11. Huallatani, 12. Lomas del Sur, 13. Apaña, 14. Cuñamani, 15. Lluto, 16. Chojo, 17. Ovejuyo, 18. Huni, 19. Tapacaya, 20. Palca, 21. Achocalla, 22. Mallasa, 23. Lipari, 24. Uma Manta, 25. Ananta, 26. Mecapaca, 27. Cota Cota, 28. El Palomar, 29. Huaricana, 30. Tahuapalca. Hábitat: bo = bosquecillos nativos de Prosopis, eu = bosques de eucaliptos (Eucalyptus globulus), ca = cardonales y matorrales xerofíticos, cu = cultivos y plantaciones de frutales, an = áreas antrópicas y urbanizadas, ac = ambientes acuáticos (lagunas y ríos), la = laderas rocosas y tierras malas (badlands), bf $=$ bofedales del altoandino, pa = pastizales y matorrales de la puna y ci $=$ cielo (aves observadas volando). Abundancia relativa según Remsen (1985): C = Común (> 10 observaciones/día), Pc = Poco común (3-10 observaciones/día), Nc = No común (1-2 observaciones/día), $\mathrm{R}=$ Raro ( $<1$ detección/día), V = Visitante (no es parte de la avifauna residente) y $\mathrm{A}=$ accidental.

\begin{tabular}{|c|c|c|c|}
\hline Especie* $^{*}(\mathrm{M}-\mathrm{E}-\mathrm{S})^{* *}$ & Localidades & Hábitat & $\begin{array}{c}\text { Abundancia } \\
\text { relativa }\end{array}$ \\
\hline \multicolumn{4}{|l|}{ TINAMIDAE } \\
\hline Nothoprocta ornata & $9,13,17,19,22,27$ & pa & Nc \\
\hline Nothoprocta pentlandii & 14,16 & pa & Nc \\
\hline \multicolumn{4}{|l|}{ ANATIDAE } \\
\hline Chloephaga melanoptera (Mov.) & 3 & bf, ac & Nc \\
\hline Lophonetta specularioides (Ma-p) & 1 & ac & Nc \\
\hline Anas flavirostris (Ma-p) & 21 & ac & $\mathrm{C}$ \\
\hline Anas georgica (Ma-e) & 21 & ac & Pc \\
\hline Anas puna (CAN) & 21 & ac & Nc \\
\hline Anas cyanoptera (Ma-p) & 21 & ac & Nc \\
\hline Oxyura jamaicensis & 21 & ac & $\mathrm{C}$ \\
\hline \multicolumn{4}{|l|}{ PODICIPEDIDAE } \\
\hline Rollandia rolland (Ma-p, CAN) & 21 & ac & Pc \\
\hline \multicolumn{4}{|l|}{ PHALACROCORACIDAE } \\
\hline Phalacrocorax brasilianus (Ma-p) & 21 & ac & $\mathrm{V}$ \\
\hline \multicolumn{4}{|l|}{ ARDEIDAE } \\
\hline Nycticorax nycticorax (Ma-p) & $21,22,25$ & ac & $\mathrm{V}$ \\
\hline Bubulcus ibis (Mb-p y Ma-p) & 21,27 & ac & $\mathrm{V}$ \\
\hline Ardea alba (Mig.) & 21 & ac & $\mathrm{V}$ \\
\hline Egretta thula (Mig.) & 21 & ac & $\mathrm{V}$ \\
\hline \multicolumn{4}{|l|}{ THRESKIORNITHIDAE } \\
\hline Plegadis ridgwayi (Mov., CAN) & 21,26 & $a c, b f$ & $\mathrm{R}$ \\
\hline \multicolumn{4}{|l|}{ CATHARTIDAE } \\
\hline Cathartes aura (Mb-p y Ma-p) & 29 & $\mathrm{ci}$ & $\mathrm{V}$ \\
\hline Vultur gryphus VUL & $9,19,26$ & ac & $\mathrm{R}$ \\
\hline \multicolumn{4}{|l|}{ ACCIPITRIDAE } \\
\hline Geranoaetus melanoleucus (Mov.) & $9,17,22,24,30$ & $\mathrm{ci}$ & $\mathrm{Nc}$ \\
\hline Buteo polyosoma (Ma-p) & $1,5,6,11,27$ & $\mathrm{ci}$ & Nc \\
\hline Buteo poecilochrous (M-alt.) & $6,9,17,18,19,22,26$ & $\mathrm{ci}$ & $\mathrm{Nc}$ \\
\hline \multicolumn{4}{|l|}{ FALCONIDAE } \\
\hline Phalcoboenus megalopterus & $1,2,5,6,9,10,12,13,14,16,17,18,21,25,26$ & ci, la & $\mathrm{C}$ \\
\hline Falco sparverius (Mb-e) & $2,4,5,6,10,12,17,18,20,21,25,26,27$ & eu, an & Pc \\
\hline Falco femoralis (Ma-e) & $6,10,11,27$ & eu, an & $\mathrm{Nc}$ \\
\hline Falco peregrinus (Mb-e y Ma-e) & $9,20,21,22$ & ac & $\mathrm{V}$ \\
\hline \multicolumn{4}{|l|}{ RALLIDAE } \\
\hline Pardirallus sanguinolentus (Ma-p) & $21,22,26,27$ & ac & $\mathrm{Nc}$ \\
\hline Gallinula chloropus (Ma-p) & 21,27 & ac & Pc \\
\hline Fulica ardesiaca (Mov.) & 21 & ac & $\mathrm{C}$ \\
\hline \multicolumn{4}{|l|}{ CHARADRIIDAE } \\
\hline Vanellus resplendens (M-alt.) & $7,21,22,25$ & ac, bf & Nc \\
\hline \multicolumn{4}{|l|}{ LARIDAE } \\
\hline Larus serranus (M-alt.) & $8,21,22,25,27$ & $\mathrm{ci}, \mathrm{ac}$ & $\mathrm{Nc}$ \\
\hline \multicolumn{4}{|l|}{ COLUMBIDAE } \\
\hline Columba maculosa (Mig.) & $6,9,21,25,26,28$ & $\mathrm{cu}, \mathrm{eu}$ & $\mathrm{Nc}$ \\
\hline Columba livia (introducido) & $2,4,6,8,9,11,13,20,21,26,27,28$ & an & $\mathrm{C}$ \\
\hline Zenaida auriculata (Ma-e) & $4,5,7,8,9,10,12,13,17,22,25,26,27,29$ & an, $\mathrm{cu}$ & $\mathrm{C}$ \\
\hline Columbina picui (Ma-e) & $7,21,22,23,25,26,27,29$ & an, $\mathrm{cu}$ & $\mathrm{C}$ \\
\hline Metriopelia ceciliae (M-alt., CAN) & $2,5,9,10,13,14,17,18,23,24,25,26,27,29,30$ & $\mathrm{la}, \mathrm{cu}$ & Pc \\
\hline Metriopelia melanoptera & $2,5,11,14,15,16,17,19,24$ & la, pa & Nc \\
\hline Leptotila megalura (CAN) & 30 & bo, $\mathrm{cu}$ & Nc \\
\hline \multicolumn{4}{|l|}{ PSITTACIDAE } \\
\hline Psilopsiagon aymara (M-alt.) & $5,6,7,8,9,18,21,22,23,25,26$ & bo, ca, cu & Pc \\
\hline Psilopsiagon aurifrons & 26 & $\mathrm{cu}$ & $\mathrm{R}$ \\
\hline
\end{tabular}


Apéndice 1. Lista de las aves del la ciudad de La Paz y regiones aledañas. (Continuación)

\begin{tabular}{|c|c|c|c|}
\hline Aratinga mitrata (Mov.) & 30 & $\mathrm{cu}, \mathrm{eu}$ & Nc \\
\hline \multicolumn{4}{|l|}{ CUCULIDAE } \\
\hline Coccyzus americanus (Mb-e) & 27 & $\mathrm{eu}, \mathrm{ac}$ & V \\
\hline \multicolumn{4}{|l|}{ TYTONIDAE } \\
\hline Tyto alba & 20,28 & an & $\mathrm{R} ?$ \\
\hline \multicolumn{4}{|l|}{ STRIGIDAE } \\
\hline Bubo virginianus & 25 & bo, ca & $\mathrm{R}$ \\
\hline Athene cunicularia & 7 & pa & $\mathrm{R}$ \\
\hline \multicolumn{4}{|l|}{ CAPRIMULGIDAE } \\
\hline Caprimulgus longirostris (Ma-p) & 24 & la & Pc \\
\hline \multicolumn{4}{|l|}{ APODIDAE } \\
\hline Aeronautes andecolus & $7,9,12,14,16,22,23,27,29$ & ci & $\mathrm{C}$ \\
\hline \multicolumn{4}{|l|}{ TROCHILIDAE } \\
\hline Colibri coruscans (M-alt.) & $2,4,5,6,8,10,17,21,22,24,25,26,27,28,30$ & bo & $\mathrm{C}$ \\
\hline Amazilia chionogaster (Ma-p) & $6,7,9,10,12,20,22,23,24,26,29$ & bo, eu & $\mathrm{Nc}$ \\
\hline Oreotrochilus estella & $1,2,3,4,5,6,9,10,12,14,17,19,21,22,27$ & pa, ac & Nc \\
\hline Patagona gigas (Ma-e) & $2,5,9,10,12,13,17,22,23,24,26,27,28,29$ & bo, ca, an & $\mathrm{C}$ \\
\hline Lesbia nuna (M-alt.) & 24 & bo & $\mathrm{R}$ \\
\hline Sappho sparganura (M-alt.) & $13,15,10,9,26,23,27,17,24,25,12,5,2,22,30,6$ & bo, ca & $\mathrm{C}$ \\
\hline \multicolumn{4}{|l|}{ PICIDAE } \\
\hline Colaptes rupicola (CAN) & $2,9,11,13,12,14,15,16,17,19,22,26,27$ & la, an, pa & $\mathrm{C}$ \\
\hline \multicolumn{4}{|l|}{ FURNARIIDAE } \\
\hline Geositta cunicularia (Ma-p) & 7 & pa & $\mathrm{R}$ \\
\hline Geositta punensis (CAN) & 23 & la & Nc \\
\hline Geositta rufipennis (Ma-p) & $9,27,26$ & pa & $\mathrm{R}$ \\
\hline Geositta tenuirostris & 14 & pa & $\mathrm{R}$ \\
\hline Upucerthia andaecola (CAN) & $5,9,10,12,13,14,16,17,25,26$ & la, $\mathrm{cu}$ & Nc \\
\hline Upucerthia ruficaudus & $23,25,27$ & la, cu & $\mathrm{R}$ \\
\hline Upucerthia harterti (endémica, CAN) & 26 & bo & $\mathrm{R}$ \\
\hline Cinclodes fuscus (Ma-p) & $2,5,6,10,11,12,13,14,16,17,19,20,21,26,27,30$ & bo & $\mathrm{C}$ \\
\hline Phleocryptes melanops (Ma-p) & 21 & ac & $\mathrm{Nc}$ \\
\hline Leptasthenura aegithaloides & $2,5,8,9,10,13,14,16,17,22,23,25,26,27$ & la, pa & Nc \\
\hline Leptasthenura fuliginiceps & $5,6,7,9,10,11,13,17,18,22,25,26,28,30$ & la, pa & Nc \\
\hline Cranioleuca henricae (endémica, CAN) & 26,29 & $\mathrm{ca}$ & $\mathrm{R}$ \\
\hline Asthenes dorbignyi & $5,6,9,10,12,13,16,18,23,25,26,27,29$ & la, ca & $\mathrm{C}$ \\
\hline Asthenes modesta & $3,14,17,21$ & pa & Pc \\
\hline Asthenes humilis (CAN) & $1,3,19$ & bf & $\mathrm{R}$ \\
\hline Asthenes sclateri (punensis) & $13,14,15,16,27$ & pa, ca & Nc \\
\hline Phacellodomus striaticeps (CAN) & $9,26,27$ & pa, ca & Nc \\
\hline \multicolumn{4}{|l|}{ RHINOCRYPTIDAE } \\
\hline Melanopareia maximiliani & 26 & $\mathrm{cu}, \mathrm{bo}, \mathrm{ca}$ & $\mathrm{R}$ \\
\hline \multicolumn{4}{|l|}{ TYRANNIDAE } \\
\hline Serpophaga munda (Ma-e) & 26,30 & bo, $\mathrm{cu}$ & $\mathrm{R}-\mathrm{V}$ \\
\hline Elaenia albiceps (Ma-e, M-alt.) & 5,26 & bo & V \\
\hline Camptostoma obsoletum (Ma-e) & 26,28 & bo & V \\
\hline Anairetes parulus (Ma-p) & $5,9,10,11,13,16,22,23,25,26,27,29,30$ & bo & Pc \\
\hline Anairetes flavirostris (Ma-p) & $5,7,26$ & $\mathrm{pa}, \mathrm{bo}, \mathrm{cu}$ & $\mathrm{R}$ \\
\hline Tachuris rubrigastra (Ma-p) & 21 & ac & $\mathrm{Pc}$ \\
\hline Pyrocephalus rubinus (Ma-e) & 21 & ac & $\mathrm{V}$ \\
\hline Ochthoeca oenanthoides (CAN) & $2,4,5,8,9,10,12,13,17,18,21,22,24,25,26,27,28$ & bo, eu & $\mathrm{C}$ \\
\hline Ochthoeca leucophrys (Ma-p, M-alt.) & $5,6,9,10,13,16,26$ & bo, eu & Nc \\
\hline Myiotheretes striaticollis (Mov.) & $8,22,27$ & la & $\mathrm{V}$ \\
\hline Muscisaxicola maculirostris (Ma-e, alt) & $5,11,12,13,17,21,24,26,27$ & la & Nc \\
\hline Muscisaxicola rufivertex (Ma-e) & $5,9,11,13,14,16,25,27$ & pa & $\mathrm{C}$ \\
\hline Muscisaxicola cinerea (Ma-e) & $9,13,17,22,23,25,26$ & bf & $\mathrm{R}$ \\
\hline Muscisaxicola grisea (CAN) & $3,17,25$ & bf & Nc \\
\hline Muscisaxicola juninensis (CAN) & $3,12,14,19,27$ & bf & Nc \\
\hline Polioxolmis rufipennis (CAN) & 14 & pa & $\mathrm{R}-\mathrm{V}$ ? \\
\hline Agriornis montana & $2,5,9,13,14,15,22$ & pa & Nc \\
\hline Knipolegus aterrimus (Ma-e, M-alt.) & $22,25,26,27,28,29$ & bo & Nc \\
\hline Hirundinea ferruginea (Ma-p) & $9,25,29$ & la & V \\
\hline Pitangus sulphuratus (Ma-p) & $7,21,26,29$ & ac & $\mathrm{V}$ \\
\hline
\end{tabular}


Apéndice 1. Lista de las aves del la ciudad de La Paz y regiones aledañas. (Continuación)

\begin{tabular}{|c|c|c|c|}
\hline Tyrannus melancholicus (Ma-e) & 26,28 & $\mathrm{cu}$ & $\mathrm{V}$ \\
\hline Tyrannus savana (Ma-e) & 23,27 & $\mathrm{eu}, \mathrm{ac}$ & $\mathrm{V}$ \\
\hline \multicolumn{4}{|l|}{ COTINGIDAE } \\
\hline Phytotoma rutila (Ma-e) & $4,5,6,7,10,13,18,16,25,26,27,28,29,30$ & bo & $\mathrm{C}$ \\
\hline \multicolumn{4}{|l|}{ HIRUNDINIDAE } \\
\hline Haplochelidon andecola (M-alt., CAN) & $6,12,19,25$ & $\mathrm{ci}, \mathrm{ac}$ & $\mathrm{V}$ \\
\hline Pygochelidon cyanoleuca (Ma-e) & $3,8,25,14,17,23,26,27$ & ci, ac & $\mathrm{C}$ \\
\hline Notiochelidon murina & 20 & ci, ac & $\mathrm{V}$ \\
\hline Hirundo rustica (Mb-e) & 21,28 & $\mathrm{ci}$, ac & $\mathrm{V}$ \\
\hline \multicolumn{4}{|l|}{ TROGLODYTIDAE } \\
\hline Troglodytes aedon & $2,4,5,7,6,9,10,17,21,22,23,26,27,28,29$ & bo, an, eu & $\mathrm{C}$ \\
\hline \multicolumn{4}{|l|}{ CINCLIDAE } \\
\hline Cinclus leucocephalus (M-alt.) & 19 & ac & $\mathrm{R}$ \\
\hline \multicolumn{4}{|l|}{ TURDIDAE } \\
\hline Turdus chiguanco & $2,4,5,9,10,14,16,17,19,20,21,22,23,25,26,27,29$ & bo, cu, an & $\mathrm{C}$ \\
\hline Turdus fuscater & $2,8,10,22,26,28,30$ & bo, eu & Pc \\
\hline Turdus amaurochalinus (Ma-e) & $7,20,24,26,28,29,30$ & $\mathrm{cu}$ & $\mathrm{C}$ \\
\hline \multicolumn{4}{|l|}{ MIMIDAE } \\
\hline Mimus dorsalis (CAN) & $7,8,9,16,22,23,25,26,27,28,29$ & bo, ca & $\mathrm{C}$ \\
\hline \multicolumn{4}{|l|}{ MOTACILLIDAE } \\
\hline Anthus correndera (Ma-p) & 1,3 & bf & $\mathrm{Nc}$ \\
\hline \multicolumn{4}{|l|}{ THRAUPIDAE } \\
\hline Conirostrum cinereum & 26 & $\mathrm{cu}$ & $\mathrm{R}$ \\
\hline Thraupis sayaca (Ma-p) & $7,21,22,26,28,29,30$ & $\mathrm{cu}, \mathrm{eu}, \mathrm{bo}$ & $\mathrm{C}$ \\
\hline Thraupis bonariensis (Ma-e) & $5,9,10,23,24,25,26,29,30$ & $\mathrm{cu}, \mathrm{bo}, \mathrm{ca}$ & Pc \\
\hline Diglossa sittoides & $22,25,26,27,30$ & bo & $\mathrm{Nc}$ \\
\hline Diglossa carbonaria (CAN, 055, 056) & $2,4,5,9,11,17,22,27,30$ & bo, an & Nc \\
\hline \multicolumn{4}{|l|}{ EMBERIZIDAE } \\
\hline Zonotrichia capensis (Ma-e) & $2,4,5,6,8,9,12,13,22,23,25,26,27,27,29$ & an & $\mathrm{C}$ \\
\hline Phrygilus punensis (CAN) & $2,3,4,5,6,9,12,13,14,16,17,18,23,25,27$ & bo, la & $\mathrm{C}$ \\
\hline Phrygilus fruticeti & $2,5,9,10,11,12,13,14,15,16,17,20,23,25,26,27$ & la & Nc \\
\hline Phrygilus plebejus & $2,3,5,9,10,12,13,14,16,17,18,25,27,30$ & pa, bo & $\mathrm{C}$ \\
\hline Phrygilus unicolor & $7,12,14,15,17,19$ & bf, pa & $\mathrm{Nc}$ \\
\hline Phrygilus alaudinus & $1,3,7$ & bf & $\mathrm{Nc}$ \\
\hline Diuca speculifera (CAN) & 1 & bf & Pc \\
\hline Idiopsar brachyurus (CAN, 056) & 20 & bf & $\mathrm{R}$ \\
\hline Poospiza boliviana (CAN, 056) & 26,29 & bo, ca & $\mathrm{R}$ \\
\hline Poospiza hypochondria & $2,5,9,13,17,21,22,23,25,26,27,29$ & bo & Nc \\
\hline Poospiza torquata (Ma-p) & 30 & $\mathrm{cu}$ & $\mathrm{R}$ \\
\hline Sicalis lutea (CAN) & $12,24,26,29$ & $\mathrm{ca}$ & $\mathrm{Nc}$ \\
\hline Sicalis uropygialis (CAN) & $3,5,13,23$ & $\mathrm{ca}$ & $\mathrm{C}$ \\
\hline Sicalis olivascens (Ma-p) & $2,4,5,9,10,12,13,17,22,23,24,25,26,27,29$ & $\mathrm{ca}$ & $\mathrm{C}$ \\
\hline Sicalis flaveola (Mov.) & 30 & $\mathrm{cu}$ & $\mathrm{R}$ \\
\hline Sporophila caerulescens (Ma-e) & 26,30 & bo & $\mathrm{R}$ \\
\hline Catamenia analis (Ma-p) & $2,5,7,10,12,13,20,21,22,23,25,26,27,28,30$ & bo & $\mathrm{C}$ \\
\hline Catamenia inornata & $5,4,11$ & pa & $\mathrm{Nc}$ \\
\hline Paroaria coronata & 27 & an & A \\
\hline \multicolumn{4}{|l|}{ CARDINALIDAE } \\
\hline Saltator aurantiirostris (Ma-e) & $5,7,9,10,13,20,21,22,23,25,26,27,28$ & bo, eu, an & $\mathrm{C}$ \\
\hline \multicolumn{4}{|l|}{ ICTERIDAE } \\
\hline Gnorimopsar chopi (Mov.) & $22,26,27$ & eu, an & Pc \\
\hline \multicolumn{4}{|l|}{ FRINGILLIDAE } \\
\hline Carduelis magellanica (M-alt.) & 9,26 & pa & $\mathrm{R}$ \\
\hline Carduelis atrata & $10,13,14,15,16,23,25,27,2,3,4,7,9,18,20,24$ & bo, pa, an & $\mathrm{C}$ \\
\hline Carduelis uropygialis (Ma-p) & $6,9,12,18,23,28$ & la, pa & Nc, V \\
\hline Carduelis xanthogastra (M-alt.) & $9,22,25,26,28,30$ & bo, la, ca & PC \\
\hline \multicolumn{4}{|l|}{ PASSERIDAE } \\
\hline Passer domesticus (introducido) & 7,26 & an & $\mathrm{Nc}$ \\
\hline
\end{tabular}

